\title{
Executive functions of children born very preterm-deficit or delay?
}

\author{
Barbara Catherine Ritter • Mathias Nelle • \\ Walter Perrig • Maja Steinlin • Regula Everts
}

Received: 20 August 2012 /Revised: 28 November 2012 / Accepted: 28 November 2012 /Published online: 18 December 2012

(C) Springer-Verlag Berlin Heidelberg 2012

\begin{abstract}
This cross-sectional study examined the performance of children born very preterm and/or at very low birth weight (VPT/VLBW) and same-aged term-born controls in three core executive functions: inhibition, working memory, and shifting. Children were divided into two age groups according to the median (young, 8.00-9.86 years; old, 9.8712.99 years). The aims of the study were to investigate whether (a) VPT/VLBW children of both age groups performed poorer than controls (deficit hypothesis) or caught up with increasing age (delay hypothesis) and (b) whether VPT/ VLBW children displayed a similar pattern of performance increase in executive functions with advancing age compared with the controls. Fifty-six VPT/VLBW children born in the cohort of 1998-2003 and 41 healthy-term-born controls were recruited. All children completed tests of inhibition (ColorWord Interference Task, Delis-Kaplan Executive Function System (D-KEFS)), working memory (Digit Span Backwards, HAWIK-IV), and shifting (Trail Making Test, Number-Letter Sequencing, D-KEFS). Results revealed that young VPT/VLBW children performed significantly poorer than the young controls in inhibition, working memory, and shifting, whereas old VPT/VLBW children performed similar
\end{abstract}

B. C. Ritter $(\triangle) \cdot$ M. Steinlin $\cdot$ R. Everts

Division of Neuropaediatrics, Development and Rehabilitation,

Children's University Hospital, Inselspital,

3010 Bern, Switzerland

e-mail: barbara.ritter@insel.ch

M. Nelle

Division of Neonatology,

Children's University Hospital, Inselspital,

3010 Bern, Switzerland

W. Perrig

Institute of Psychology, University of Bern,

3012 Bern, Switzerland

W. Perrig $\cdot$ M. Steinlin $\cdot$ R. Everts

Center for Cognition, Learning and Memory,

University of Bern, Bern, Switzerland to the old controls across all three executive functions. Furthermore, the frequencies of impairment in inhibition, working memory and shifting were higher in the young VPT/ VLBW group compared with the young control group, whereas frequencies of impairment were equal in the old groups. In both VPT/VLBW children and controls, the highest increase in executive performance across the ages of 8 to 12 years was observed in shifting, followed by working memory, and inhibition. Conclusions: This study provides evidence that (a) poor performance in inhibition, working memory, and shifting of young VPT/VLBW children might reflect a delay rather than a deficit and (b) that VPT/VLBW children are likely to display a similar pattern of performance increase in these three executive functions compared with that of controls.

Keywords Children · Preterm · Working memory · Inhibition $\cdot$ Shifting $\cdot$ Catch-up

$\begin{array}{ll}\text { Abbreviations } \\ \text { VPT/ } & \text { Born very preterm or at very low birth } \\ \text { VLBW } & \text { weight } \\ \text { EF } & \text { Executive functions } \\ \text { WM } & \text { Working memory }\end{array}$

\section{Introduction}

In the current literature, it is unclear whether children born very preterm (VPT; $<32$ gestational weeks) and/or at very low birth weight (VLBW; $<1,500 \mathrm{~g}$ ) perform poorer in cognitive function tests than same-aged controls throughout childhood (deficit) or catch-up in performance with increasing age (delay). In the case of a delay, the performance difference between VPT/VLBW children and same-aged controls would be large in younger children but negligible in older children, indicating that performance differences decline with increasing age. In the case of a deficit, the performance level of both 
young and old VPT/VLBW children would continuously remain below that of same-aged controls. Some studies have reported that preterm-born children catch-up in cognitive performance over time [31, 41], whereas other studies have observed stable deficits [20] or even an accentuation of problems with increasing age [40]. To our knowledge, no evidence of catch-up has yet been described regarding executive functions (EF). To the contrary, VPT/VLBW children are reported to be at risk of deficiencies in EF across several ages [3, 4].

EF refer to cognitive processes that are important for purposeful and future-orientated behavior [26]. Miyake and colleagues [32] established three core EF, inhibition, working memory (WM), and shifting, which provide a basis for the execution of more complex executive skills such as planning or problem-solving. EF have shown to be crucial for academic achievement such as reading and writing competence [1] and mathematics [9]. The emergence of EF has been linked to structural maturation of the frontal cortex [10]. The age period of 8 to 12 years is characterized by a growth spurt in the frontal lobes, and, therefore, is a critical period where these three functions develop and become established [2].

Inhibition is described as the ability to suppress pre-potent responses, stop an ongoing process and avoid cognitive interference [32]. VPT/VLBW children at the ages of 5 to 12 years perform poorer than full-term controls in inhibition tasks compared with same-aged controls $[4,8,16]$. Poor inhibition skills in VPT/VLBW children have been linked to cerebral lesions (e.g., haemorrhage) [21] and white matter abnormalities (e.g., reduction of white matter volume, ventricular dilatation, thinning of the corpus callosum, and delay in myelination) [15].

WM refers to the ability to store and manipulate information during a short period of time [18, 27]. VPT/VLBW children are at risk of a decreased WM capacity in the preschool period [8, 42], during school years [3], and in adolescence [6]. Poor WM is likely to correspond to brain injury in the neonatal period [42].

Shifting is the ability to deliberately shift between mental tasks or sets [32]. Successful shifting taps both inhibition and WM resources, as the irrelevant set has to be inhibited while constantly monitoring whether the shifting rules are being observed [5, 32]. Furthermore, shifting displays the most prolonged developmental timetable among the three functions $[12,25,32]$. For that reason, shifting is suggested to be more complex than inhibition and WM [5]. Some studies have reported deficiencies in shifting of younger $[8,15,39]$ and older VPT/VLBW children $[4,40]$, as well as young VPT/VLBW adults [41]. In other studies, equal performance levels of shifting have been found in VPT/ VLBW and control children [37]. Inconsistencies might reflect the extent to which the instruments used to assess shifting require WM and inhibition abilities [5]. An impairment in shifting can relate to neonatal circumstances, with children being born at extremely or very low birth weight $[39,40]$ or with neonatal cerebral lesions [35] showing more problems in shifting than term-born controls.

In general, a catch-up in cognitive performance of VPT/ VLBW children is suggested to depend on (a) the degree of prematurity, with those children born earlier and at lower birth weight displaying higher frequencies of cognitive impairment $[16,39]$, (b) the extent of severe medical complications during the neonatal period, which have been linked to poorer outcome (e.g., haemorrhage, periventricular leukomalacia, necrotising enterocolitis) $[17,35]$, and (c) the parental socioeconomic milieu [28]. A study by Luu and colleagues [28] revealed that VPT/VLBW children who showed a catch-up in cognitive performance had low rates of neonatal complications and neurosensory impairment, highly educated mothers and were members of the ethnic majority. Overall, about $70 \%$ of all VPT/VLBW children born in Switzerland do not exhibit severe neonatal complications and neurosensory impairment, which increases the possibility of normal cognitive functions later in development [36].

The present study examined performance of VPT/ VLBW children and healthy term-born controls in inhibition, WM, and shifting. All children were assessed at 8 to 12 years of age, a developmental period in which the three EF become increasingly established [2]. Children were divided into two age groups according to the median (young and old). The aims of the study were to investigate whether (a) VPT/VLBW children of both age groups performed poorer than controls (deficit hypothesis) or caught up with increasing age (delay hypothesis) and (b) whether VPT/VLBW children displayed a similar pattern of performance increase in EF with advancing age compared with the controls. As low rates of neonatal complications and neurodevelopmental impairment (IQ $\geq$ 85) have been shown to be a crucial factor facilitating a catch-up in preterm-born children [28], only VPT/VLBW children with no or minimal neonatal cerebral brain lesions and no or minimal neurodevelopmental impairment were included, which represent the major subgroup of the VPT/VLBW population [36].

\section{Methods}

This study reports on a subset of data from the NEMO research program (NEuropsychology and meMOry) at the Children's University Hospital in Bern, Switzerland. The NEMO project examines cognitive performance in VPT/VLBW children and same-aged term-born controls in a cross sectional study design. All children completed the same cognitive assessment tasks. The study protocol has been approved by the ethics committee of the Children's University Hospital in Bern. All children and caregivers provided informed written consent prior to 
participation, consistent with the Code of Ethics of the World Medical Association (Declaration of Helsinki).

\section{Participants}

$V P T / V L B W$ sample The medical records of all surviving German-speaking VPT/VLBW children born in the cohort of 1998 to 2003 at the Children's University Hospital in Bern, Switzerland, were reviewed. Inclusion criteria of the NEMO research program were: (a) born at $<32$ weeks gestational age and/or $<1,500 \mathrm{~g}$ birth weight, (b) currently aged between 7 and 12 years, (c) no or mild neonatal cerebral lesions (maximal hemorrhage grade II), (d) no or mild periventricular leukomalacia (maximal grade II), (e) no chronic illness potentially influencing development (e.g., birth deformity, congenital heart defect, cerebral palsy, and epilepsy), (f) no postnatally occurring medical problems potentially influencing development (e.g., meningitis, encephalopathy, and traumatic brain injury), (g) no pervasive developmental disorders (e.g., autism), (h) IQ $\geq 85$ in neuropsychological assessment, and (i) normal or corrected-to-normal vision and hearing. Children fulfilling these inclusion criteria exhibit low rates of neonatal complications and neurodevelopmental impairment, and are considered to be the ones most likely to catch-up on executive performance of term-born controls [28]. According to the medical records, 247 children ( $92.5 \%$ of the screened sample) fulfilled inclusion criteria. They were contacted by a letter including an information booklet for parents and children. Seventy-five children $(29.1 \%$ of the children fulfilling the inclusion criteria) agreed to enter the study and completed the neuropsychological assessment, but three children had to be excluded because of IQ $<85(n=2 ; 2.7 \%)$ or refusal $(n=1$; $1.3 \%$ ). A total of 72 VPT/VLBW children remained. Two of the EF tests (Delis-Kaplan Executive Function System (DKEFS)) used in this study require sophisticated reading ability and knowledge of the alphabetical order (see "Assessment"), that is why the 7-year-old VPT/VLBW children were excluded from analyses. The remaining 8- to 12-year olds were divided into two age groups according to the median of the VPT/ VLBW and the control sample (young, 8.00-9.86 years; old, 9.87-12.99 years). Furthermore, VPT/VLBW children of both age groups were selected as being comparable for IQ and maternal SES. Finally, a total of 56 VPT/VLBW children (28 young and 28 old children; 26 girls and 30 boys) was included. The frequencies of neonatal complications and therapies of these children are reported in Table 1.

Control sample Healthy term-born German-speaking children were recruited with announcements on notice boards in the hospital and local schools. Inclusion criteria were: (a) born $>37$ weeks of gestation and $>2,500 \mathrm{~g}$ birth weight, (b) aged between 7 and 12 years, (c) no chronic illness potentially influencing development (e.g., birth deformity, congenital heart defect, cerebral palsy, and epilepsy), (d) no medical problems potentially influencing development (e.g., meningitis, encephalopathy, and traumatic brain injury), (e) no pervasive developmental disorders (e.g., autism), (f) IQ $\geq 85$ in the neuropsychological assessment, and (g) normal or correctedto-normal vision and hearing. Fifty-three children completed the neuropsychological assessment, but three $(5.7 \%)$ were excluded due to $\operatorname{ADHD}(n=2 ; 3.8 \%)$ or refusal during testing $(n=1 ; 1.9 \%)$. No child had to be excluded due to IQ $<85$. Overall, 50 controls remained. Two of the EF tests (D-KEFS) used in this study require sophisticated reading ability and knowledge of the alphabetical order (see "Assessment"), that is why the 7-year-old controls were excluded from analyses. The remaining 8- to 12-year olds were divided into two age groups according to the median of the VPT/VLBW and the control sample (young, 8.00-9.86 years; old, 9.8712.99 years). Furthermore, controls of both age groups were selected as being comparable for IQ and maternal SES. Finally, a total of 41 control children ( 21 young and 20 old children; 21 girls and 20 boys) was included.

\section{Assessment}

To assess performance in inhibition, WM, and shifting, well-known clinical neuropsychological tests were used. The children's raw scores were transformed into agecorrected scaled scores according to the respective test manuals. A high-scaled score indicates good performance, whereas a low scaled score indicates poor performance. Impairment was defined as scaled score of $<7$.

Inhibition was measured using the D-KEFS Color-Word Interference [13]. This test consists of four conditions, whereas the third condition is the primary task of inhibition. The child has to name the ink color of the words that are printed in an incongruent ink (e.g., the child has to name the color (red) in which a word (green) is printed). The variable of interest was commission errors, which occur when the child reads the word instead of naming the color. A highscaled score indicates that the child made few commission errors and, therefore, had good inhibition skills.

As a measure of WM, the backwards digit span task of the German Version of the "Wechsler intelligence scale for children," fourth edition, was used (HAWIK-IV) [33, 42]. The variable of interest was the maximal backward span attained. A high-scaled score indicates that the child achieved a high backward span and, therefore, had good WM capacity.

Shifting was assessed using the forth condition of the D-KEFS Trail Making Test (number-letter switching) [13]. In this condition, the child had to shift between two sets of stimuli (sets A and B) by means of connecting numbers (set A, 1 to 16) and letters (set B, A to P), switching between connecting numbers and letters in correct order as fast as 
Table 1 Neonatal characteristics and therapies of the VPT/VLBW children

\begin{tabular}{lll}
\hline Young VPT/ & Old VPT/ & All VPT/ \\
VLBW children & VLBW children & VLBW children \\
\hline
\end{tabular}

Gestational age (weeks)

$\begin{array}{ll}\text { Median } & 29.9 \\ \text { Range } & 26.7-33.6 \\ \text { Gestational age (<28 weeks) } & \end{array}$

$\begin{array}{lll}n & 7 & 6 \\ \% & 25\end{array}$

$\% \quad 25$

Birth weight (g)

Median 1,180

Range $\quad 890-1,870$

Birth weight $(<1,000 \mathrm{~g})$

$n \quad 8$

$\% \quad 28.6$

\section{4}

$25.8-33.7$

30.0

$25.7-33.7$

Intrauterine growth restriction $^{\mathrm{a}}$

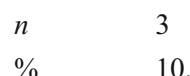

10.7

Prenatal steroids (complete) ${ }^{\mathrm{b}}$

$$
\begin{array}{ll}
n & 13 \\
\% & 46.4
\end{array}
$$

Prenatal steroids (incomplete) ${ }^{\mathrm{c}}$

$\begin{array}{lll}n & 5\end{array}$

$\% \quad 17.9$

Postnatal steroids ${ }^{\mathrm{d}}$

$\begin{array}{ll}n & 4 \\ \% & 14.3\end{array}$

\section{5}

Surfactant therapy ${ }^{\mathrm{e}}$

$\begin{array}{ll}n & 5 \\ \% & 17.9\end{array}$

Chronic lung disease ${ }^{\mathrm{f}}$

n 7

$\% \quad 25$

Necrotizing enterocolitis ${ }^{\mathrm{g}}$

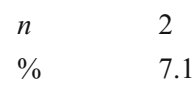

Sepsis ${ }^{\mathrm{h}}$

$n \quad 1$

$\% \quad 3.6$

Hemorrhage grade I-II

$\begin{array}{ll}n & 2 \\ \% & 7.1\end{array}$

$\%$ - 7.1

7.1

1

Periventriuclar leukomalacia grade $\mathrm{I}-\mathrm{II}^{\mathrm{j}}$

$\begin{array}{llll}n & 0 & 0 & 0 \\ \% & 0 & 0 & 0\end{array}$

Patent ductus arteriosus ${ }^{\mathrm{k}}$

$\begin{array}{lc}n & 2 \\ \% & 7.1 \\ \text { Remedial teaching } & \\ n & 4\end{array}$

$n \quad 4$
6

21.4

\section{1,200}

$570-2,060$

7

25

17.9

17

60.7

5
17.9

5

17.9

\section{6}

21.4

3

10.7

13

23.2

1,190

$570-2,060$

15

26.8

8

14.3

30

53.6

10

17.9

9

16.1

11

19.6

10

17.9

4

7.1

3

5.4

6

10.7

0

0

6

$\begin{array}{ll}14.3 & 10.7\end{array}$

$4 \quad 8$
Table 1 (continued)

\begin{tabular}{|c|c|c|c|}
\hline & $\begin{array}{l}\text { Young VPT/ } \\
\text { VLBW children }\end{array}$ & $\begin{array}{l}\text { Old VPT/ } \\
\text { VLBW children }\end{array}$ & $\begin{array}{l}\text { All VPT/ } \\
\text { VLBW children }\end{array}$ \\
\hline$\%$ & 14.3 & 14.3 & 14.3 \\
\hline \multicolumn{4}{|c|}{ Occupational therapy ${ }^{1}$} \\
\hline$n$ & 5 & 3 & 8 \\
\hline$\%$ & 17.9 & 10.7 & 14.3 \\
\hline \multicolumn{4}{|c|}{ Speech therapy ${ }^{1}$} \\
\hline$n$ & 3 & 2 & 5 \\
\hline$\%$ & 10.7 & 7.1 & 8.9 \\
\hline
\end{tabular}

${ }^{a}$ Birth weight below the 10th percentile for gestational age

${ }^{\mathrm{b}}$ At least two doses of steroids, with $24 \mathrm{~h}$ in between, last dose at $>24 \mathrm{~h}$ before birth

${ }^{\mathrm{c}}$ Only one dose of steroids or last dose at $<24 \mathrm{~h}$ before birth

${ }^{\mathrm{d}}$ At least one dose

e At least one dose, any product

${ }^{\mathrm{f}}$ Need of oxygen $>28$ days after birth

${ }^{g}$ Clinical signs (abdominal distention, bilious aspirates and/or bloody stools) and confirmed by X-ray intramural gas or at laparatomy

${ }^{\mathrm{h}}$ Proven through clear clinical, radiological, or histological evidence of infection as well as at least on microbiologically relevant positive blood culture

${ }^{\mathrm{i}}$ Assessed by cerebral ultrasound within first $24 \mathrm{~h}$, at days 3 and 7 and then every 1 or 2 weeks until final discharge [29]

${ }^{\mathrm{j}}$ Periventricular hyperechogenicity lasting for more than 7 days

${ }^{\mathrm{k}}$ Symptomatic, requiring indomethacin, ibuprofen, or surgery

${ }^{1}$ Ongoing

possible (i.e., 1-A-2-B-3-C, and so on). The variable of interest was the time (in seconds) needed to complete the task. A high scaled score indicates that the child needed little time to connect the numbers and letters in correct order and, therefore, had good shifting skills. To control the shifting performance for the basic processing speed attained in only one set of stimuli, condition two (number sequencing; set A, 1 to 16) was administered.

General intelligence was measured using the short form of the German version of the HAWIK-IV [11, 33, 42]. Fullscale IQ was calculated from seven subtests of the original HAWIK-IV (block design, similarities, digit span, coding, vocabulary, matrix reasoning, and symbol search) [11].

Maternal socioeconomic status (SES) has been suggested to have a greater impact on cognitive outcome of pretermborn children than paternal SES $[28,41]$. Maternal SES was defined as the mother's education level at the time of the neuropsychological assessment (no graduation $=1$, college $=$ 2 , college of higher education $=3$, and university degree $=4$ ). Ethnic majority status was defined as having a Swiss passport or German as first family language.

Information about gestational age, birth weight and neonatal complications of the VPT/VLBW children was collected from the children's neonatal medical records. 


\section{Procedure}

After written consent from the caregivers was obtained, the participants were contacted by telephone to set a date for the neuropsychological assessment. Children were tested individually by trained neuropsychologists (R.E. and B.R.). All tasks were administered in a standardized order as part of a larger neuropsychological battery. Children were offered regular breaks.

\section{Statistical analyses}

All statistical analyses were performed using the Statistical Package for Social Sciences software for Windows, version 17 (SPSS, Chicago, Illinois). In order to decide which statistical methods fitted best to the data, all continuous variables were tested for normal distribution using Kolmogorov-Smirnov test (age, IQ, maternal SES, and the performance in inhibition, WM, and shifting). Because the majority of the variables were not normally distributed, non-parametric statistical test methods were further applied (Kolmogorov-Smirnov test results-IQ, $p=0.09$; age, $p<$ 0.05; maternal SES, $p<0.01$; inhibition, $p<0.01$; WM, $p<$ 0.01 ; and shifting, $p<0.01$ ).

First, it was tested whether the VPT/VLBW and the control sample were comparable regarding demographic variables (sex, ethnic majority status, age, IQ, and maternal SES; see "Analyses of demographic data"). One-tailed Pearson's Chi-square tests were conducted for categorical variables (sex and ethnic majority status) and one-tailed MannWhitney $U$ tests were computed for continuous variables (age, IQ, and maternal SES). It was tested whether the whole VPT/VLBW and the whole control sample were comparable regarding age, IQ, and maternal SES (all-all). Comparison of these variables was also conducted including the young groups of both samples (young-young) and the old groups of both samples separately (old-old) (see Table 2).

Second, it was tested whether EF performance (scaled scores) differed significantly between the young VPT/ VBLW group and the young control group as well as between the old VPT/VLBW group and the old control group (see "Group differences, effect sizes, and frequencies of impairment"). For this purpose, Mann-Whitney $U$ tests were conducted. To disclose the performance differences beyond $p$ values, effect sizes were calculated (Cliff's delta) [24]. A Cliff's delta near to 1.0 or -1.0 indicates only a small overlap between VPT/VLBW children and controls (large difference between groups), while a Cliff's delta near to 0.0 indicates a large overlap between the two groups (small difference between groups) [24]. In case of a deficit, both young and old VPT/VLBW groups would perform significantly poorer than the respective control group (i.e., large effect size). In case of a delay, the young VPT/VLBW group would perform inferior to the young control group (i.e., large effect size), whereas the old VPT/VLBW group would perform similarly to the old control group (i.e., small effect size). Furthermore, it was tested whether the frequencies of EF impairment (scaled score of $<7$ ) differed between the young VPT/VBLW group and the young control group as well as between the old VPT/VLBW group and the old control group using one-tailed Pearson's Chi-square tests.

Third, it was tested whether the VPT/VLBW sample and the control sample showed significant correlations between age and EF performance (raw scores). For this purpose, Spearman's correlation coefficients were calculated. A significant correlation indicates that the EF performance markedly increased with age, whereas a nonsignificant correlation reveals that the children already reached a quite mature level of performance. Raw scores (and not scaled scores) were chosen because scaled scores are age-corrected and, therefore, should ideally not correlate with age.

\section{Results}

Analyses of demographic data

The results of the following analyses are reported in Table 2. There was no difference in sample size between the VPT/ VLBW age groups and the control age groups $(p=0.403)$. Sex and ethnic background were equally distributed in the VPT/VLBW and the control sample (sex, $p=0.320$; ethnic majority, $p=0.325$ ).

Both VPT/VLBW age groups were comparable to the respective control age groups in terms of age (young, $p=$ 0.201; old, $p=0.425$ ) but not regarding IQ (young, $p<0.01$; old, $p<0.01$ ) and maternal SES (young, $p<0.01$; old, $p<$ 0.01 ), with the controls having significant higher IQs and maternal SES compared with the VPT/VLBW children.

Group differences, effect sizes, and frequencies of impairment

The results of the following analyses are reported in Table 3. The effect sizes are shown in Fig. 1.

Inhibition Young VPT/VLBW children performed significantly lower in the inhibition task than young controls $(p<$ $0.05)$, whereas old VPT/VLBW children performed similarly to old controls $(p=0.409)$. Effect sizes were small-tomedium between the young groups $(d=0.30)$ and negligible between the old groups $(d=0.06)$. Pearson's Chi-square test revealed significantly higher frequency of inhibition impairment in the young VPT/VLBW group compared with the young control group $(p<0.01)$. No difference in the 


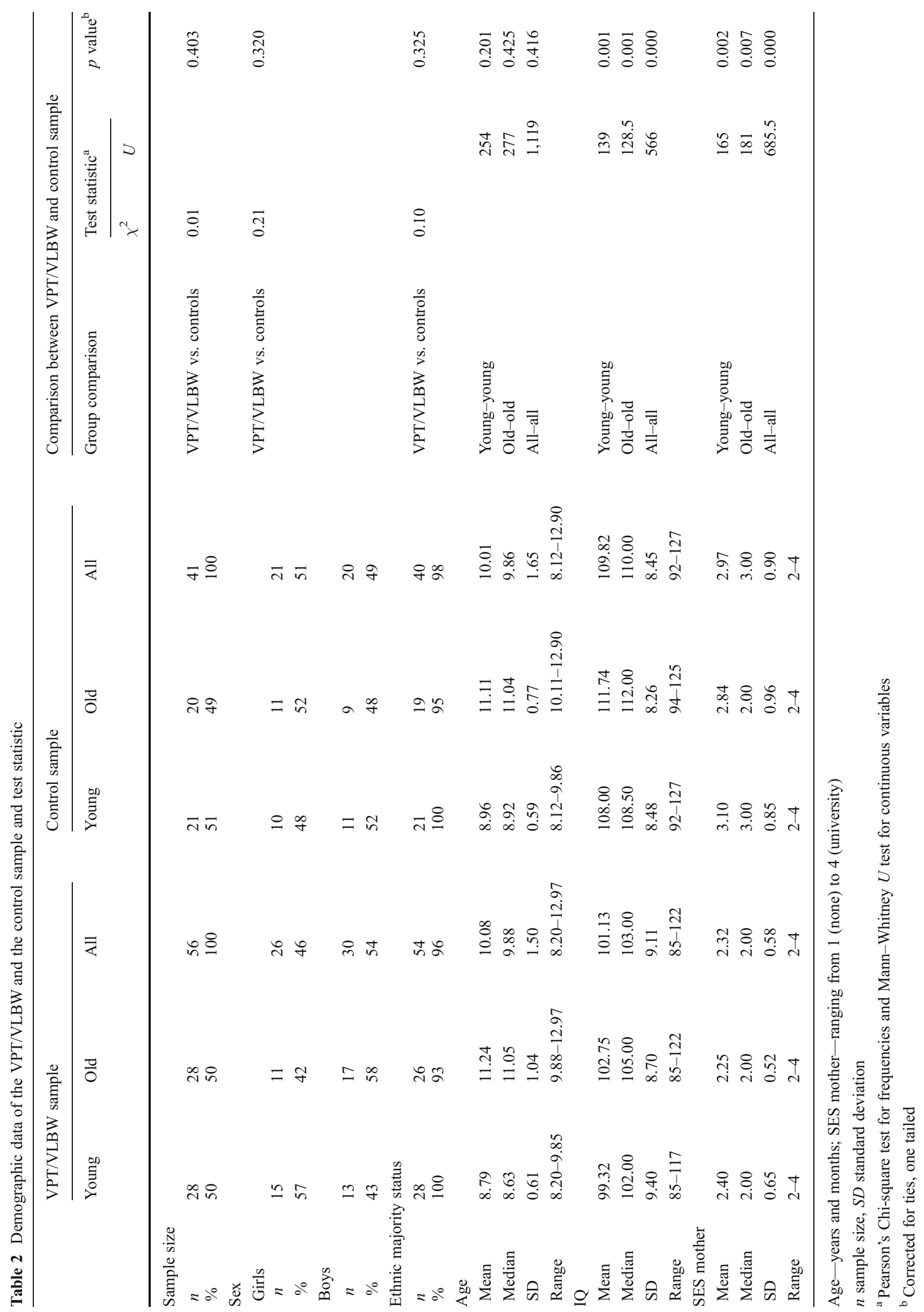


Table 3 Comparisons between young and old VPT/VLBW children and controls, effect sizes, and percentages of impaired children

\begin{tabular}{|c|c|c|c|c|c|c|c|c|c|c|}
\hline \multirow[t]{2}{*}{ Statistics } & \multicolumn{2}{|l|}{ Sample } & \multicolumn{2}{|l|}{ Test statistic } & \multirow{2}{*}{$\begin{array}{l}\text { Effect size } \\
\text { Cliff's delta }\end{array}$} & \multicolumn{5}{|l|}{ Test statistic } \\
\hline & $\begin{array}{l}\text { VPT/VLBW } \\
\text { children }\end{array}$ & Controls & $\begin{array}{l}\text { Mann-Whitney } \\
U \text { test }\end{array}$ & $p$ value $^{\mathrm{a}}$ & & Group & $n$ & $\%$ & $\chi^{2}$ & $p$ value $^{\mathrm{b}}$ \\
\hline \multicolumn{11}{|l|}{ Inhibition } \\
\hline \multicolumn{11}{|c|}{ Young children } \\
\hline Mean & 9.36 & 10.75 & 205.5 & 0.035 & 0.30 & VPT/VLBW vs. controls & 7 & 25 & 6.13 & 0.007 \\
\hline Median & 10.00 & 11.00 & & & & & 0 & 0 & & \\
\hline $\mathrm{SD}$ & 3.00 & 1.41 & & & & & & & & \\
\hline Range & $2-14$ & $9-13$ & & & & & & & & \\
\hline \multicolumn{11}{|c|}{ Old children } \\
\hline Mean & 9.71 & 9.68 & 255.5 & 0.409 & 0.06 & VPT/VLBW vs. controls & 2 & 7 & 0.27 & 0.303 \\
\hline Median & 9.50 & 10.00 & & & & & 2 & 10 & & \\
\hline SD & 3.00 & 2.48 & & & & & & & & \\
\hline Range & $6-14$ & $3-13$ & & & & & & & & \\
\hline \multicolumn{11}{|c|}{ Working memory } \\
\hline \multicolumn{11}{|c|}{ Young children } \\
\hline Mean & 10.12 & 10.70 & 196.5 & 0.028 & 0.31 & VPT/VLBW vs. controls & 0 & 0 & $--^{\mathrm{c}}$ & $-^{\mathrm{c}}$ \\
\hline Median & 10.00 & 10.50 & & & & & 0 & 0 & & \\
\hline SD & 1.67 & 1.34 & & & & & & & & \\
\hline Range & $7-12$ & $8-13$ & & & & & & & & \\
\hline \multicolumn{11}{|c|}{ Old children } \\
\hline Mean & 9.43 & 9.60 & 235.5 & 0.165 & 0.13 & VPT/VLBW vs. controls & 0 & 0 & $--^{\mathrm{c}}$ & $--^{\mathrm{c}}$ \\
\hline Median & 9.50 & 10.00 & & & & & 0 & 0 & & \\
\hline SD & 1.10 & 1.70 & & & & & & & & \\
\hline Range & $7-12$ & $7-12$ & & & & & & & & \\
\hline \multicolumn{11}{|l|}{ Shifting } \\
\hline \multicolumn{11}{|c|}{ Young children } \\
\hline Mean & 8.12 & 11.05 & 126.5 & $0.001^{\mathrm{d}}$ & 0.49 & VPT/VLBW vs. controls & 8 & 29 & 2.87 & 0.045 \\
\hline Median & 9.00 & 12.00 & & & & & 2 & 10 & & \\
\hline SD & 3.75 & 2.49 & & & & & & & & \\
\hline Range & $2-14$ & $6-15$ & & & & & & & & \\
\hline \multicolumn{11}{|c|}{ Old children } \\
\hline Mean & 10.00 & 11.05 & 174 & $0.021^{\mathrm{e}}$ & 0.35 & VPT/VLBW vs. controls & 2 & 10 & 1.42 & 0.117 \\
\hline Median & 10.00 & 11.00 & & & & & 0 & 0 & & \\
\hline SD & 2.70 & 1.70 & & & & & & & & \\
\hline Range & $2-14$ & $8-14$ & & & & & & & & \\
\hline
\end{tabular}

$n$ sample size, $S D$ standard deviation

${ }^{a}$ Corrected for ties, one tailed

${ }^{\mathrm{b}}$ One tailed

${ }^{\mathrm{c}}$ Pearson's Chi-square test cannot be computed because the frequencies of impairment are zero in both groups of both samples

${ }^{\mathrm{d}}$ Remained significant when accounting for basic processing speed $(p<0.05)$

${ }^{\mathrm{e}}$ Data no longer significant when accounting for processing speed $(p=0.723)$

frequency of inhibition impairment occurred in the old groups $(p=0.303)$.

Working memory Young VPT/VLBW children performed significantly lower in the WM task than young controls $(p$ $<0.05$ ), whereas old VPT/VLBW children performed similarly to old controls $(p=0.165)$. Effect sizes were small-to-medium between the young groups $(d=0.31)$ and small between the old groups $(d=0.13)$. Frequencies of WM impairment were zero in both the VPT/VLBW and control group and, therefore, no Pearson's Chisquare test was computed. 
Fig. 1 The effect sizes of group differences between VPT/

VLBW children and controls

\section{Effect sizes}

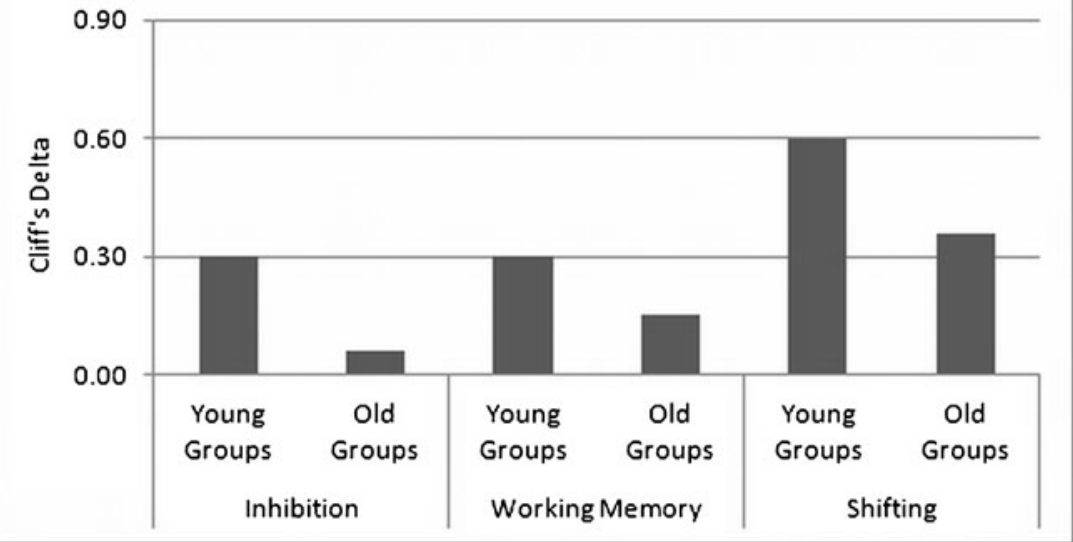

Shifting Young VPT/VLBW performed significantly lower in the shifting task than young controls $(p<$ $0.01)$. The difference remained significant when accounting for basic processing speed $(p<0.05)$. Old VPT/VLBW children performed significantly lower in the shifting task than old controls $(p<0.05)$ but the difference was no longer significant when accounting for basic processing speed $(p=0.723)$. Effect sizes were small-to-medium between the young groups $(d=$ 0.49 ) and small-to-medium between the old groups $(d=0.35)$. Pearson's Chi-square test revealed significant higher frequency of shifting impairment in the young VPT/VLBW group compared with the young control group $(p<0.05)$. No difference in the frequency of shifting impairment occurred between the old VPT/ VLWB and the old control group $(p=0.117)$.

\section{Correlation between age and EF performance}

The Spearman's correlation coefficients are reported in Table 4. In the VPT/VLBW sample, there was a significant correlation between age and inhibition performance $(r=-0.30$, $p<0.01)$, WM performance $(r=0.39, p<0.01)$, and shifting performance $(r=-0.64, p<0.01)$. In the control sample, there was a significant correlation between age and WM performance $(r=0.39, p<0.01)$ and shifting performance $(r=-0.68$, $p<0.01)$. No correlation occurred between age and inhibition performance $(r=-0.12, p=0.231)$.

\section{Discussion}

The first aim of the study was to investigate whether VPT/ VLBW children performed poorer than controls in both age groups (deficit hypothesis) or caught up with increasing age (delay hypothesis). Regarding inhibition performance, young
VPT/VLBW children performed significantly poorer than young controls, indicating that young VPT/VLBW children committed more errors in the inhibition task than young controls. In contrast, inhibition performance of old VPT/VLBW children and old controls was similar. Concordantly, the frequency of inhibition impairment was significantly higher in the young VPT/VLBW group than the young control group but similar in the old groups.

Regarding WM performance, young VPT/VLBW children performed significantly poorer compared with young controls, indicating that young VPT/VLBW children displayed shorter backwards digit span than young controls. In contrast, WM performance of old VPT/VLBW children and old controls was similar. The frequencies of WM impairment were zero in both the VPT/VLBW and the control sample, revealing that all children performed above the threshold of clinical impairment. This result is not surprising

Table 4 Spearman's correlation coefficients between age and executive function performance in VPT/VLBW children and controls

\begin{tabular}{llc}
\hline Sample & Executive function & Spearman's rho \\
\hline VPT/VLBW children & Inhibition & $-0.30^{*}$ \\
& Working memory & $0.39^{*}$ \\
& Shifting & $-0.64^{\mathrm{a}, *}$ \\
Controls & Inhibition & $-0.12 \mathrm{n} . \mathrm{s}$. \\
& Working memory & $0.39^{*}$ \\
& Shifting & $-0.68^{\mathrm{b}, *}$ \\
\hline
\end{tabular}

Spearman's correlation coefficients between age and the VPT/VLBW and control children's raw scores in the tests of inhibition, working memory, and shifting are reported.

n.s. nonsignificant

$* p<0.01$

${ }^{\mathrm{a}}$ Remained significant when accounting for processing speed $(p<$ $0.001)$

${ }^{\mathrm{b}}$ Remained significant when accounting for processing speed $(p<0.01)$ 
when considering the nature of the WM task. Span tasks do usually not produce large performance variances because there is a small window of possible results (e.g., a backward span of 2, 3, 4, or 5), which increases the possibility of performance within the normal range.

Regarding shifting performance, young VPT/VLBW children performed significantly poorer compared with young controls, even when accounting for basic processing speed. This result indicates that young controls needed significantly more time to complete the shifting task compared with the young controls, independent of basic processing speed. Old VPT/VLBW children performed significantly poorer in the shifting task compared with old controls, however, the difference was no longer significant when accounting for basic processing speed. This result indicates that old VPT/VLBW children performed poorer than old controls because of slower processing speed rather than shifting problems. Concordantly, the frequency of shifting impairment was significantly higher in the young VPT/VLBW group compared with the young control group, but similar in the old groups.

Together, the present data suggest a decrease of performance difference between VPT/VLBW children and controls with increasing age. These results support the delay hypothesis, which is plausible when considering the characteristics of the study sample: VPT/VLBW children exhibited no or minimal neonatal brain lesions, no or mild neurodevelopmental impairment, well-educated mothers and are mainly members of the ethnic majority, all factors that facilitate a catch-up of cognitive performance in VPT/ VLBW children [28]. The hypothesis of delay is further strengthened when bearing in mind that controls had significantly higher IQs and maternal SES and, therefore, reflected a high-functioning and economically favored group. Despite lower IQ and SES, old VPT/VLBW children managed to produce performance levels comparable to those of controls across all three EF.

The support of the delay hypothesis in VPT/VLBW children is contradictory to the literature, where VPT/ VLBW children usually performed significantly poorer in EF tests compared with controls [3, 4, 8]. However, studies commonly compare the performance of VPT/VLBW children as a collective to a control group rather than dividing them into different age groups, enabling a more differentiated look at the course of EF development. When comparing the performance of collectives with large age ranges, a delay in EF performance might remain undetected because the lower performance of the younger children contributes to the significant group difference. This bias might even be more accentuated in studies including VPT/VLBW children under the age of 9 years, where a possible catch-up might not yet have occurred. Accordingly, it is desirable that future studies on VPT/VLBW children address more attention to a possible delay in order to avoid misleadingly labeling older VPT/VLBW children as inferior compared with same-aged controls.

Catch-up in cognitive functions is likely to be underpinned by structural and/or functional cerebral maturation and environmental effects. The emergence of executive skills has been linked to structural maturation of the frontal lobes, in particular of the prefrontal cortex [10]. A spurt of grey matter growth in the prefrontal cortex occurs at about the age of 11 years in girls and 12 years in boys [19]. The old VPT/VLBW children in this study were about to undergo this period of crucial structural brain development. Difficulties in EF seen at younger ages might vanish at an older age, possibly due to prolonged maturation of the frontal brain structures. A study by Kesler and colleagues [23] revealed that by the age of 12 years, brain morphology and cognitive performance of VPT/VLBW girls with no neonatal cerebral brain lesions was similar to that of same-aged term-born females. As mentioned above, catch-up may be additionally underpinned by functional maturation of the brain, more precisely by the emergence of compensatory mechanisms. It is possible that VPT/VLBW children compensate for reduced regional cerebral volume [34] by recruiting supplementary brain regions to achieve the same level of performance as controls. Accordingly, VPT/VLBW children at 12 years of age were observed to activate different functional networks during a language processing task than controls, despite similar task performance [30]. Furthermore, catch-up might depend on environmental factors. Special care, such as remedial teaching, and occupational or speech therapy, was needed in $9-18 \%$ of the VPT/VLBW children. These interventions are likely to affect VPT/VLBW children by means of a performance increase in trained skills and, hence, an approximation to the performance levels of healthy controls. Together, the mechanisms underpinning catch-up remain unknown and should be the subject of future research.

Several studies have shown that VPT/VLBW adults still exhibit impairment in EF compared with term-born adults [6, 41], supporting the deficit hypothesis. However, VPT/VLBW adults were born at times when there was a different standard of neonatal care. In recent years, neonatal intensive-care medicine has strongly improved, for example due to antenatal steroid and postnatal surfactant therapy [38]. The present study sample was born between 1998 to 2003, a period where the use of antenatal steroids and surfactant was already embedded in the usual treatment of these children. Overall, it is conceivable that a catch-up in cognitive performance occurs more frequently in VPT/VLBW children born only recently because these children can benefit from modern medical intensive care standards. 
The second aim of this study was to investigate whether VPT/VLBW children displayed a similar pattern of performance increase in EF with advancing age compared with controls. In the VPT/VLBW sample, age correlated significantly with performance in inhibition, WM, and shifting. These results indicate that, with increasing age, VPT/VLBW children committed less inhibition errors, showed higher backwards digit span, and needed less time to shift between two sets of stimuli. These findings correspond to several studies including 8 - to 12 -year-old healthy children $[12,18]$. In the present study, the highest performance increase (i.e., the largest correlation) was observed in shifting, followed by WM, and, at last, by inhibition. This result is in line with the literature suggesting that shifting skills rely on both inhibition and WM resources and, therefore, demonstrate the most prolonged developmental timetable among the three EF [5, 12].

In the control sample, age correlated significantly with WM and shifting, but not with inhibition. These results indicate that, with increasing age, controls showed higher backwards digit span and needed less time to shift between two sets of stimuli, but did not commit less inhibition errors, indicating that controls already reached a quite mature level of performance regarding inhibition. The highest performance increase was observed in shifting, followed by $\mathrm{WM}$, and, at last, by inhibition. This sequence indicates that inhibition was already more matured compared with WM and shifting, which is in line with another study, where healthy children aged 8 to 13 years showed significant performance increases in WM and shifting, but not in inhibition [25]. Diamond [14] suggested the ability to resist interference and reliably inhibit inappropriate responses to be fundamental for the emergence of higher-order performance. In other words, as long as irrelevant stimuli cannot be suppressed efficiently, processing relevant information is error-prone. Similarly, Bjorklund and Harnishfeger [7] considered successful inhibition skills to increase the efficiency of WM, insofar as efficient inhibition hinders irrelevant information from entering the WM operation pool. Together, the VPT/ VLBW children showed a widely similar pattern of performance increase in executive skills compared with controls, with highest performance increase in shifting, followed by WM, and inhibition.

There are a few limitations to be mentioned. First, the study design was cross-sectional. Final Conclusions about delay or deficit best derive from studies with longitudinal designs. Second, the sample size of each age group was rather small, which decreases the power to detect significant differences between the groups and increases the chance of outliers distorting the results [22]. Given the importance of inhibition, $\mathrm{WM}$, and shifting for academic achievement, longitudinal studies with large sample sizes are needed to give further insight into the development of EF in VPT/VLBW children. Third, the VPT/VLBW cohort was non-representative because only children with no or minimal brain lesions and no or minimal neurodevelopmental impairment were included. The current results are not comparable to those of studies on preterm-born children with more severe neonatal complications, which are thought to have higher rates of cognitive dysfunction $[15,42]$. In future research, it would be of interest to investigate EF performance in VPT/VLBW children with different severities of neonatal course and different histories of neurodevelopmental impairment.

Together, this study provides evidence for the delay hypothesis and suggests that VPT/VLBW children can catch-up in performance of inhibition, WM, and shifting with increasing age. Furthermore, VPT/VLBW children showed a similar pattern of performance increase in these three functions with advancing age, with the highest performance increase in shifting, followed by WM, and inhibition. In conclusion, poor performance in inhibition, WM and shifting of young VPT/VLBW children with no or minimal brain lesions and no or minimal neurodevelopmental impairment might reflect a delay rather than a deficit.

Acknowledgments We thank the Swiss National Neonatal FollowUp Group for their collaboration. The research was funded by the Swiss National Science Foundation (PZ00P1_126309).

\section{References}

1. Altemeier L, Jones J, Abbott RD, Berninger VW (2006) Executive functions in becoming writing readers and reading writers: note taking and report writing in third and fifth graders. Dev Neuropsychol 29:161-173. doi:10.1207/s15326942dn2901_8

2. Anderson P (2002) Assessment and development of executive function (EF) during childhood. Child Neuropsychol 8:71-82. doi:10.1076/chin.8.2.71.8724

3. Anderson PJ, Doyle LW (2004) Executive functioning in schoolaged children who were born very preterm or with extremely low birth weights in the 1990s. Pediatrics 114:50-57. doi:10.1542/ peds.114.1.50

4. Bayless S, Stevenson J (2007) Executive functions in school-age children born very prematurely. Early Hum Dev 83:247-254. doi:10.1016/j.earlhumdev.2006.05.021

5. Best JR, Miller PH (2010) A developmental perspective on executive function. Child Dev 81:1641-1660. doi:10.1111/j.14678624.2010.01499.x

6. Bhutta AT, Cleves MA, Casey PH, Cradock MM, Anand KJ (2002) Cognitive and behavioral outcomes of school-aged children who were born preterm: a meta-analysis. JAMA 288:728-737. doi:10.1001/jama.288.6.728

7. Bjorklund DF, Harnishfeger KK (1990) The resources construct in cognitive development: diverse sources of evidence and a theory of inefficient inhibition. Dev Rev 10:48-71. doi:10.1016/0273-2297 (90)90004-N

8. Böhm B, Smedler AC, Forssberg H (2004) Impulse control, working memory and other executive functions in preterm children when starting school. Acta Paediatr 93:1363-1371. doi:10.1111/ j.1651-2227.2004.tb02938.x 
9. Bull R, Scerif G (2001) Executive functioning as a predictor of children's mathematics ability: inhibition, switching, and working memory. Dev Neuropsychol 19:273-293. doi:10.1207/ S15326942DN1903 3

10. Casey BJ, Giedd JN, Thomas KM (2000) Structural and functional brain development and its relation to cognitive development. Biol Psychol 54:241-257. doi:10.1016/S0301-0511(00)00058-2

11. Crawford JR, Anderson V, Rankin PM, MacDonald J (2010) An index-based short-form of the WISC-IV with accompanying analysis of the reliability and abnormality of differences. Br J Clin Psychol 49:235-258. doi:10.1348/014466509X455470

12. Davidson MC, Amso D, Anderson LC, Diamond A (2006) Development of cognitive control and executive functions from 4 to 13 years: evidence from manipulations of memory, inhibition, and task switching. Neuropsychologia 44:2037-2078. doi:10.1016/j.neuropsychologia.2006.02.006

13. Delis DC, Kaplan E, Kramer J (2001) Delis-Kaplan executive function system. The Psychological Corporation, San Antonio

14. Diamond A (1991) Frontal lobe involvement in cognitive changes during the first year of life. In: Gibson KR, Petersen AC (eds) Brain maturation and cognitive development: comparative and cross-cultural perspectives. de Gruyter, New York, pp 127-180

15. Edgin JO, Inder TE, Anderson PJ, Hood KM, Clark CA, Woodward LJ (2008) Executive functioning in preschool children born very preterm: relationship with early white matter pathology. J Int Neuropsychol Soc 14:90-101. doi:10.1017/S1355617708080053

16. Elgen I, Lundervold AJ, Sommerfelt K (2004) Aspects of inattention in low birth weight children. Pediatr Neurol 30:92-98. doi:10.1016/S0887-8994(03)00402-8

17. Farel AM, Hooper SR, Teplin SW, Henry MM, Kraybill EN (1998) Very-low-birthweight infants at seven years: an assessment of the health and neurodevelopmental risk conveyed by chronic lung disease. J Learn Disabil 31:118-126. doi:10.1177/002221949803100202

18. Gathercole SE, Pickering SJ, Ambridge B, Wearing H (2004) The structure of working memory from 4 to 15 years of age. Dev Psychol 40:177-190. doi:10.1037/0012-1649.40.2.177

19. Giedd JN, Blumenthal J, Jeffries NO, Castellanos FX, Liu H, Zijdenbos A, Paus T, Evans AC, Rapoport JL (1999) Brain development during childhood and adolescence: a longitudinal MRI study. Nat Neurosci 2:861-863. doi:10.1038/13158

20. Johnson S, Fawke J, Hennessy E, Rowell V, Thomas S, Wolke D et al (2009) Neurodevelopmental disability through 11 years of age in children born before 26 weeks of gestation. Pediatrics 124:249257. doi: $10.1542 /$ peds.2008-3743

21. Katz KS, Dubowitz LMS, Henderson S, Jongmans M, Kay GG, Nolte CA, de Vries L (1996) Effect of cerebral lesions on continuous performance test responses of school age children born prematurely. $\mathrm{J}$ Pediatr Psychol 21:841-855. doi:10.1093/jpepsy/21.6.841

22. Keppel G, Wickens TD (2004) Design and analysis: a researcher's handbook (4th edition). Prentice-Hall, Upper Saddle River

23. Kesler SR, Reiss AL, Vohr B, Watson C, Schneider KC, Katz KH et al (2008) Brain volume reduction within multiple cognitive systems in male preterm children at age twelve. J Pediatr 152:513-520. doi:10.1016/j.jpeds.2007.08.009

24. Ledesma RD, Macbeth G, Cortada de Kohan N (2009) Computing effect size measures with ViSta - the visual statistics system. Tutor Quant Methods Psychol 5:25-34

25. Lehto JE, Juujärvi P, Kooistra L, Pulkkinen L (2003) Dimensions of executive functioning: evidence from children. $\mathrm{Br} \mathrm{J}$ Dev Psychol 21:59-80. doi:10.1348/026151003321164627

26. Lezak M (1995) Neuropsycholocial assessment (3rd edn). Oxford University Press, New York
27. Luciana M, Conklin HM, Hooper CJ, Yarger RS (2005) The development of nonverbal working memory and executive control processes in adolescents. Child Dev 76:697-712. doi:10.1111/ j.1467-8624.2005.00872.x

28. Luu TM, Vohr BR, Allan W, Schneider KC, Ment L (2011) Evidence for catch-up in cognition and receptive vocabulary among adolescents born very preterm. Pediatrics 128:313-322. doi:10.1542/peds.2010-1421

29. Ment LR, Bada HS, Barnes P, Grant PE, Hirtz D, Papile LA, PintoMartin J, Rivkin M, Slovis TL (2002) Practice parameter: neuroimaging of the neonate: report of the Quality Standards Subcommittee of the American Academy of Neurology and the Practice Committee of the Child Neurology Society. Neurology 58:1726-1738. doi:10.1212/WNL.58.12.1726

30. Ment LR, Peterson BS, Vohr B, Allan W, Schneider KC, Lacadie C et al (2006) Cortical recruitment pattern in prematurely-born children compared to controls during a passive listening fMRI task. J Pediatr 149:490-498. doi:10.1016/j.jpeds.2006.06.007

31. Ment LR, Vohr B, Allan W, Katz KH, Schneider KC, Westerveld $M$ et al (2003) Change in cognitive function over time in very lowbirth-weight infants. JAMA 289:705-711. doi:10.1001/ jama.289.6.705

32. Miyake A, Friedman NP, Emerson MJ, Witzki AH, Howerter A, Wager TD (2000) The unity and diversity of executive functions and their contributions to complex "Frontal Lobe" tasks: a latent variable analysis. Cogn Psychol 41:49-100. doi:10.1006/ cogp.1999.0734

33. Petermann F, Petermann U (2008) Hamburg-WechslerIntelligenztest für Kinder-IV (HAWIK-IV). Huber, Bern

34. Peterson B, Vohr B, Staib LH, Cannistraci CJ, Dolberg A, Schneider KC et al (2000) Regional brain volume abnormalities and long-term cognitive outcome in preterm infants. JAMA 284:1939-1947. doi:10.1001/jama.284.15.1939

35. Ross G, Boatright S, Auld PA, Nass R (1996) Specific cognitive abilities in 2-year-old children with subependymal and mild intraventricular hemorrhage. Brain Cogn 32:1-13. doi:10.1006/ brcg.1996.0054

36. Rüegger C, Hegglin M, Adams M, Bucher U (2012) Population based trends in mortality, morbidity and treatment for very preterm and very low birth weight infants over 12 years. BMC Pediatr. doi:10.1186/1471-2431-12-17

37. Rushe TM, Rifkin L, Stewart AL, Townsend JP, Roth SC, Wyatt JS et al (2001) Neuropsychological outcome at adolescence of very preterm birth and its relation to brain structure. Dev Med Child Neurol 43:226-233. doi:10.1111/j.1469-8749.2001.tb00194.x

38. Saigal S, Doyle LW (2008) An overview of mortality and sequelae of preterm birth from infancy to adulthood. Lancet 371:261-269. doi:10.1016/S0140-6736(08)60136-1

39. Taylor HG, Klein N, Schatschneider C, Hack M (1998) Predictors of early school age outcomes in very low birth weight children. J Dev Behav Pediatr 19:235-243

40. Taylor HG, Minich NM, Klein N, Hack M (2004) Longitudinal outcomes of very low birth weight: neuropsychological findings. J Int Neuropsychol Soc 10:149-163. doi:10.1017/ S1355617704102038

41. Tideman E (2000) Longitudinal follow-up of children born preterm: cognitive development at age 19. Early Hum Dev 58:81-90. doi:10.1016/S0378-3782(00)00055-4

42. Woodward LJ, Edgin JO, Thompson D, Inder TE (2005) Object working memory deficits predicted by early brain injury and development in the preterm infant. Brain 128:2578-2587. doi:10.1093/brain/awh618 